\title{
PRODUÇÃO DE MUDAS DE Albizia lebbeck (L.) Benth SOB DIFERENTES FORMULAÇÕES E DOSES DE OSMOCOTE ${ }^{\circledR}$
}

\section{SEEDLING PRODUCTION OF Albizia lebbeck (L.) Benth UNDER DIFFERENT OSMOCOTE ${ }^{\circledR}$ FORMULATIONS AND DOSES}

\author{
Marcos Vinícius Miranda Aguilar ${ }^{1}$, Marília Dutra Massad², Tiago Reis Dutra ${ }^{3}$, \\ Eduarda Soares Menezes, Aline Ramalho dos Santos ${ }^{5}$, Fabiano Guimarães Silva ${ }^{6}$ \\ ${ }^{1}$ Universidade Federal de Santa Maria, Santa Maria, RS, Brasil-aguilarmarcos2009@hotmail.com \\ 2, 3, 6 Instituto Federal de Educação, Ciência e Tecnologia do Norte de Minas Gerais, Campus Salinas, \\ Salinas, MG, Brasil - mariliamassad@yahoo.com.br, tiagoreisdutra@gmail.com \&
} fabiano1976@yahoo.com.br

${ }^{4}$ Universidade Federal dos Vales do Jequitinhonha e Mucuri, Diamantina, MG, Brasil eduarda_menezs@hotmail.com

${ }^{5}$ Universidade Federal do Espírito Santo, Jerônimo Monteiro, ES, Brasil - alineramalho13@hotmail.com

\section{RESUMO}

O objetivo do trabalho foi avaliar a produção e a qualidade de mudas de Albizia lebbeck (L.) Benth em resposta à aplicação de diferentes formulações e doses de Osmocote ${ }^{\circledR}$. Foi adotado o delineamento experimental em blocos casualizados, com três repetições, no esquema fatorial $3 \times 5$, sendo estudado o efeito de três formulações de Osmocote ${ }^{\circledast}$ (Osmocote ${ }^{\circledR}$ MiniPrill Controlled Realise 19-06-10 com liberação de 3 a 4 meses; Osmocote ${ }^{\circledR}$ Plus (com Micro-Nutriente) 15-09-12 com liberação de 5 a 6 meses; Osmocote ${ }^{\circledR}$ Plus (com Micro-Nutriente) 15-09-12 com liberação de 7 a 8 meses, e cinco dosagens dos mesmos (0; 2,5; 5,0; 7,5 e 10,0 g dm${ }^{-3}$ ). Cada unidade experimental foi constituída por 12 mudas. Foram avaliados aos 120 dias à altura $(\mathrm{H} ; \mathrm{cm})$, diâmetro do coleto $(\mathrm{DC}, \mathrm{mm})$, matéria seca da parte aérea (MSPA; g planta-1), matéria seca da raiz (MSR; g planta-1) e matéria seca total $(M S T=M S P A+$ MSR; g planta-1 ${ }^{1}$ ), além das relações H/MSPA, MSPA/MSR e Índice de Qualidade de Dickson (IQD). O Osmocote ${ }^{\circledR}$ com $^{\circ}$ tempo de liberação de 7 a 8 meses proporciona maior produção de massa seca às mudas de albízia. A maior dose estudada $\left(10,0 \mathrm{~g} \mathrm{dm}^{-3}\right)$ permitiu as maiores médias para as variáveis analisadas, entretanto, os valores foram semelhantes à dose $5,0 \mathrm{~g} \mathrm{dm}^{-3}$, o que permite uma economia nos custos de produção. Os adubos Osmocote ${ }^{\circledR}$ com $^{\circ}$ tempo de liberação 5 a 6 meses, 7 a 8 meses e 3 a 4 meses são recomendados para a produção de mudas de albízia.

PALAVRAS-CHAVE: Adubo de liberação lenta, Albízia, Silvicultura.

\section{ABSTRACT}

The objective of this work was to evaluate the production and quality of Albizia lebbeck (L.) Benth seedlings in response to the application of different formulations and doses of Osmocote ${ }^{\circledR}$. A randomized complete block design with three replications was adopted in a $3 \times 5$ factorial scheme and the effect of three formulations of Osmocote ${ }^{\circledR}$ (Osmocote ${ }^{\circledR}$ Plus (with Micro Nutrient) 15-09-12 with release of 5 to 6 months, Osmocote ${ }^{\circledR}$ Plus (with MicroNutrient) 15-09-12 with release from 7 to 8 months, and Osmocote ${ }^{\circledR}$ MiniPrill Controlled Realize 19-06-10 with release from 3 to 4 months and five dosages $\left(0 ; 2.5 ; 5.0 ; 7.5\right.$ and $\left.10.0 \mathrm{~g} \mathrm{dm}^{-3}\right)$ Each experimental unit consisted of 12 seedlings. At 120 days height $(\mathrm{H} ; \mathrm{cm})$, stem diameter $(\mathrm{DC}, \mathrm{mm})$, shoot dry matter (MSPA; g plant ${ }^{-1}$ ), root dry matter (MSR; g plant $\left.{ }^{-1}\right)$ and weight were evaluated total drought $\left(\mathrm{MST}=\mathrm{MSPA}+\mathrm{MSR}\right.$; $\left.\mathrm{g} \mathrm{plant}^{-1}\right)$, in addition to $\mathrm{H}$ / MSPA, MSPA / MSR ratios and Dickson Quality Index (IQD). Osmocote ${ }^{\circledR}$ with a release time of 7 to 8 months provides higher dry mass production for albízia seedlings. The highest dose studied $\left(10.0 \mathrm{~g} \mathrm{dm}^{-3}\right)$ allowed the highest averages for the analyzed variables, however, the values were similar to the $5.0 \mathrm{~g} \mathrm{dm}^{-3}$ dose, which allows for savings in production costs. Osmocote ${ }^{\circledR}$ fertilizers with release time of 5 to 6 months, 7 to 8 months and 3 to 4 months are recommended for the production of albízia seedlings.

KEYWORDS: Slow release fertilizer, Albízia, Forestry. 


\section{INTRODUÇÃO}

O conhecimento relacionado ao cultivo de espécies florestais tem se desenvolvido bastante com o aumento da demanda por implantação florestal para diversas finalidades, desde comerciais ou ecológicas. Nesse contexto, desponta-se a necessidade da recomposição de ecossistemas degradados, sendo necessário o aprimoramento de tecnologias, viabilidade técnica e econômica em projetos para a produção de mudas (MENEGATTI et al., 2017).

Existem diversas espécies que podem ser utilizadas nas atividades de reflorestamento, dentre elas está a Albizia lebbeck (L.) Benth (1844), popularmente conhecida como albízia. Nativa da Ásia tropical, a espécie é caracterizada por apresentar um rápido crescimento, habilidade para fixar nitrogênio e melhorar a estrutura do solo, especialmente em áreas degradadas, possuindo usos múltiplos e facilidade para consórcio com culturas agrícolas (DUTRA \& MEDEIROS FILHO, 2009).

Projetos voltados para restauração florestal e a busca por espécies com alta capacidade de adaptação expandem-se em mesmo ritmo implicando em maior necessidade de estudos que utilizem o desenvolvimento de mudas com alta qualidade, e simultaneamente custos menos elevados (MEWS et al., 2015).

$\mathrm{O}$ conhecimento relacionado aos fatores que afetam o desenvolvimento das plantas é essencial para melhorar as técnicas de viveiricultura (TRAUTENMULLE et al., 2017b). Entretanto, o sucesso da produção de mudas florestais nos viveiros muitas vezes é limitado pela falta de informações quanto às demandas nutricionais das espécies. Desse modo, conhecer as necessidades nutricionais de uma espécie permite produzir mudas de melhor qualidade, bem como plantas que se adaptam com maior facilidade a campo, e com maior índice de sobrevivência (DUTRA et al., 2016).

As práticas de adubação favorecem o desenvolvimento das mudas, e dessa forma diminui 0 tempo de permanência das plantas no viveiro, reduzindo assim os custos de produção (CHEN et al., 2018). A aplicação de fertilizantes que possuem liberação lenta ou ação controlada de nutrientes permite uma disponibilidade contínua do mesmo, evitando o uso parcelado de algumas fontes, e diminuindo os custos de operação (GIBSON et al., 2019).

O Osmocote ${ }^{\circledR}$ é um fertilizante que libera nutrientes de maneira controlada. É formado por cápsulas com nutrientes envoltos por uma resina orgânica biodegradável, que assegura proteção, contudo disponíveis por pressão osmótica, assim se concede a liberação, podendo variar de intensidade, conforme a umidade e temperatura (ALMEIDA et al., 2018). Dadas às suas características, existe menor possibilidade de ocorrer deficiência de nutrientes durante o período de formação das mudas, o que confere sua grande capacidade para produção das mesmas (ROSA et al., 2018).

Entretanto, estudos que avaliem o efeito de adubos de liberação lenta em espécies com potencial paisagístico e de recuperação de áreas degradadas ainda são incipientes (ROSSA et al., 2015a). Assim, torna-se necessário ampliar as pesquisas sobre a reposta das espécies florestais a diferentes formulações e doses de Osmocote ${ }^{\circledR}$ a fim de se determinar as formas de manejo das mudas.

Diante do exposto, o presente trabalho teve como objetivo avaliar a produção e a qualidade de mudas de albízia (Albizia lebbeck (L.) Benth) em resposta à aplicação de diferentes formulações e doses de Osmocote ${ }^{\circledR}$.

\section{MATERIAL E MÉTODOS}

\section{Área de estudo}

O experimento foi realizado no "Viveiro de Produção de Mudas Florestais" do Instituto Federal de Educação, Ciência e Tecnologia do Norte de Minas Gerais (IFNMG), Câmpus Salinas.

O município de Salinas encontra-se localizado entre $16^{\circ} 10^{\prime} 19^{\prime \prime}$ de latitude Sul, e $42^{\circ} 17^{\prime} 30^{\prime \prime}$ de longitude Oeste, e está situado na mesorregião do Norte de Minas possuindo altitude média de $471 \mathrm{~m}$. O clima da região, de modo geral, varia de subúmido a semiárido, classificado majoritariamente, segundo Köppen, como sendo Aw, de inverno seco e verão chuvoso (TOLEDO et al., 2009; MENEZES et al., 2019).

\section{Condução do experimento}

Foi adotado o delineamento experimental em blocos casualizados, com três repetições, no esquema fatorial $3 \mathrm{x}$ 5 , sendo estudado o efeito de três formulações de Osmocote ${ }^{\circledast}$ (Osmocote ${ }^{\circledR}$ MiniPrill Controlled Realise 1906-10 com liberação de 3 a 4 meses); Osmocote ${ }^{\circledR}$ Plus (com Micro-Nutriente) 15-09-12 com liberação de 5 a 6 meses; Osmocote ${ }^{\circledR}$ Plus (com Micro-Nutriente) 15-09-12 com liberação de 7 a 8 meses) e o e cinco dosagens dos mesmos $\left(0 ; 2,5 ; 5,0 ; 7,5\right.$ e $\left.10,0 \mathrm{~g} \mathrm{dm}^{-3}\right)$. Cada unidade experimental foi constituída por 12 mudas.

As sementes de albízia foram coletadas de sete árvores matrizes localizadas no município de Araçuaí-MG. 
O substrato utilizado para produção das mudas foi o Rohrbacher $^{\circledR}$, cuja caracterização química foi realizada conforme descrito por Empresa Brasileira de Pesquisa Agropecuária (EMBRAPA) (1997). As características físicas de porosidade total, macroporosidade, microporosidade, e capacidade máxima de retenção de água dos substratos foram determinadas por metodologia proposta por Carvalho \& Silva (1992) (Tabela 1).

Tabela 1. Características químicas e físicas do substrato utilizado na produção de mudas de Albizia lebbeck (L.) Benth

\begin{tabular}{|c|c|}
\hline \multirow{2}{*}{ Características $^{1}$} & Substrato \\
\hline & Rohrbacher $^{\circ}$ \\
\hline pH, água & 6,0 \\
\hline M.O, dag kg-1 & 15,63 \\
\hline $\mathrm{P}, \mathrm{mg} \mathrm{dm^{-3 }}$ & 260,00 \\
\hline $\mathrm{K}, \mathrm{mg} \mathrm{dm^{-3 }}$ & 970 \\
\hline $\mathrm{Ca}, \mathrm{cmol}_{\mathrm{c}} \mathrm{dm}^{-3}$ & 5,10 \\
\hline $\mathrm{Mg}, \mathrm{cmol}_{\mathrm{c}} \mathrm{dm}^{-3}$ & 1,60 \\
\hline $\mathrm{Al}, \mathrm{cmol}_{\mathrm{c}} \mathrm{dm}^{-3}$ & 0,00 \\
\hline $\mathrm{H}+\mathrm{Al}, \mathrm{cmol}_{\mathrm{c}} \mathrm{dm}^{-3}$ & 2,19 \\
\hline $\mathrm{t}, \mathrm{cmol}_{\mathrm{c}} \mathrm{dm}^{-3}$ & 9,19 \\
\hline $\mathrm{T}, \mathrm{cmol}_{\mathrm{C}} \mathrm{dm}^{-3}$ & 11,38 \\
\hline $\mathrm{SB}, \mathrm{cmol}_{\mathrm{C}} \mathrm{dm}^{-3}$ & 9,19 \\
\hline $\mathrm{m}, \%$ & 0 \\
\hline $\mathrm{V}, \%$ & 81 \\
\hline Porosidade Total, \% & 61,19 \\
\hline Macroporosidade, \% & 31,43 \\
\hline Microporosidade, \% & 29,76 \\
\hline CMRA, $\mathrm{mL} 55 \mathrm{~cm}^{-3}$ & 23,11 \\
\hline
\end{tabular}

${ }^{1}$ M.O. = matéria orgânica; $\mathrm{t}=$ capacidade efetiva de troca de cátions; $\mathrm{T}$ = capacidade de troca de cátions; $\mathrm{SB}=$ soma de bases; $\mathrm{m}$ = saturação por alumínio; $\mathrm{V}=$ saturação por bases; $\mathrm{CMRA}=$ Capacidade máxima de retenção de água.

Para superar a impermeabilidade do tegumento das sementes, foi realizada a superação da dormência por meio do método da escarificação mecânica utilizando lixa d'água número 120 até desgastar o tegumento no lado oposto ao da micrópila (DUTRA et al., 2007). Em seguida, foram desinfestadas em solução de hipoclorito de sódio (2\%) por 3 minutos.

O substrato foi preparado de forma que incorporou ao mesmo as doses e formulações de Osmocote ${ }^{\circledR}$ estudadas. Após o preparo do substrato, o mesmo foi destinado para o preenchimento dos tubetes de $180 \mathrm{~cm}^{3}$, sendo esses identificados e destinados ao local de estudo. Após esse procedimento, ocorreu a semeadura, utilizando três sementes por tubete. Aos 15 dias após semeadura (DAS) efetuou-se um primeiro raleio deixando-se duas plantas por tubete. Aos 30 DAS um segundo raleio foi realizado, deixando-se apenas uma muda por tubete.

A partir do 40으, as mudas receberam fertirrigação semanal, com $6 \mathrm{~mL}$ planta-1 de solução aquosa, contendo $4 \mathrm{~g} \mathrm{~L}^{-1}$ de sulfato de amônio, $10 \mathrm{~g} \mathrm{~L}^{-1}$ de superfosfato simples, $4 \mathrm{~g} \mathrm{~L}^{-1}$ de cloreto de potássio e $1 \mathrm{~g} \mathrm{~L}^{-1}$ de FTE BR12 ( $9 \% \mathrm{Zn}, 3 \% \mathrm{Fe}, 2 \% \mathrm{Mn}, 0,1 \% \mathrm{Mo}, 1,8 \% \mathrm{~B}, 0,8 \% \mathrm{Cu}$ ). Durante o período experimental, a umidade do solo foi mantida próxima de $60 \%$ da capacidade de campo procedendo-se o monitoramento diário para esse controle.

\section{Parâmetros avaliados}

Foram avaliados aos 120 dias à altura $(\mathrm{H} ; \mathrm{cm})$ e o diâmetro do coleto (DC; $\mathrm{mm}$ ) das mudas. A mensuração da altura da parte aérea das mudas foi realizada com o auxílio de uma régua milimétrica posicionada no nível do solo até o meristema apical das mesmas, e o diâmetro com o uso de um paquímetro digital.

Ao final dessas avaliações, as plantas foram colhidas e separadas em parte aérea e sistema radicular, lavadas em água corrente e secas em estufa com circulação forçada de ar a aproximadamente 65 으, até peso constante. Avaliou-se a matéria seca da parte aérea (MSPA; g planta$\left.{ }^{1}\right)$, matéria seca da raiz (MSR; g planta-1) e matéria seca total $\left(\mathrm{MST}=\mathrm{MSPA}+\mathrm{MSR} ; \mathrm{g}\right.$ planta $\left.{ }^{-1}\right)$. Esses parâmetros foram transformados em índices de qualidade de mudas conforme sugerido por Gomes et al. (2002): H/DC, H/MSPA, MSPA/MSR e no Índice de Qualidade de Dickson - IQD (DICKSON et al., 1960).

$$
I Q D=\frac{M S T(g)}{\frac{H(\mathrm{~cm})}{D C(\mathrm{~mm})}+\frac{M S P A(\mathrm{~g})}{M S R(\mathrm{~g})}}
$$

Os resultados obtidos foram submetidos à análise de variância e ao ser constatada a significância pelo teste $F$, as médias dos tratamentos foram comparadas pelo teste Tukey a $5 \%$ de probabilidade por meio do pacote ExpDes.pt (FERREIRA et al., 2013) do software livre $R$ ( $R$ CORE TEAM, 2015).

\section{RESULTADOS E DISCUSSÃO}

Houve efeito significativo da interação entre os fatores avaliados (formulações e doses de Osmocote ${ }^{\circledR}$ ) para as variáveis altura da parte aérea e diâmetro do coleto (Tabela 2). 
Tabela 2. Valores médios de altura da parte aérea e diâmetro do coleto de mudas de albízia (Albizia lebbeck (L.) Benth) em resposta a diferentes formulações e doses de Osmocote ${ }^{\circledR}$ aos 120 dias



Para a variável altura da parte aérea os osmocotes com tempo de liberação 5 a 6 meses e 7 a 8 meses, a partir da dose 5,0 $\mathrm{g} \mathrm{dm}^{-3}$, apresentaram médias estatisticamente iguais, entretanto, superiores ao Osmocote 3 a 4 meses (Tabela 2). A formulação 7 a 8 meses apresentou médias similares nas doses 7,5 e 10,0 g $\mathrm{dm}^{-3}$, enquanto os demais osmocotes apresentaram esse comportamento entre suas doses a partir de 5,0 $\mathrm{g} \mathrm{dm}^{-3}$. Esse resultado evidencia que as formulações de Osmocote 5 a 6 meses e 7 a 8 meses garantiram a manutenção de um sincronismo entre a liberação de nutrientes ao longo do tempo e o atendimento às necessidades nutricionais da albízia com diferentes doses cada um, permitindo uma maior potencialidade de crescimento das mudas da espécie.

A altura é uma característica relevante quando há a necessidade do plantio de mudas em áreas colonizadas por plantas espontâneas, onde a altura da espécie de interesse precisa exercer maior competição por luz. Em diversos viveiros florestais, a altura é levada em consideração para determinar o valor das mudas na ocasião da comercialização ao silvicultor, sendo comumente usada para estimar seu padrão de qualidade (GOMES et al., 2002; SOMAVILLA et al., 2014). Dessa forma, a variável constitui um dos parâmetros importantes para estimar o crescimento no campo, visto que sua medição não causa destruição, apresenta fácil execução, e tecnicamente é aceita como uma excelente medida do potencial de desempenho das mudas (ROSSA et al., 2015b).
Resultados semelhantes ao deste trabalho foram observados por Massad et al. (2016) trabalhando com mudas das espécies flamboyant e ipê-mirim em resposta a diferentes doses de Osmocote ${ }^{\circledR}$ e por Marana et al. (2008), avaliando a qualidade das mudas de café, onde a dose aproximada de $15 \mathrm{~g} \mathrm{dm}^{-3}$ de fertilizante de liberação lenta apresentou as maiores médias para a variável altura, medida 150 dias após a semeadura. Elli et al. (2013) observaram comportamento semelhante com Osmocote ${ }^{\circledR}$ de 5 a 6 meses na produção de mudas de pitangueira e Stupp et al. (2015) trabalhando com mudas de bracatinga em função de diferentes doses de fertilizante de liberação lenta.

Para o diâmetro do coleto as formulações de Osmocote ${ }^{\circledR}$ com liberação de 5 a 6 meses e 7 a 8 meses se igualaram estatisticamente a partir da dose $2,5 \mathrm{~g} \mathrm{dm}^{-3}$, apresentando médias superiores ao Osmocote ${ }^{\circledR} 3$ a 4 meses (Tabela 2).

Esse resultado pode ser atribuído ao fato dessas formulações proporcionarem melhores condições para as mudas de albízia com o fornecimento de nutrientes de maneira regular, contemplando um período maior, atendendo a demanda da espécie e favorecendo o desenvolvimento da muda.

A partir da dose de $5,0 \mathrm{~g} \mathrm{dm}^{-3}$, não houve ganho significativo de diâmetro de coleto para os osmocotes 5 a 6 meses e 7 a 8 meses. Esse comportamento foi observado para o Osmocote ${ }^{\circledR} 3$ a 4 meses a partir de 7,5 g $\mathrm{dm}^{-3}$.

Resultados positivos mediante a aplicação de adubos de liberação lenta para a variável diâmetro do coleto foram evidenciados em estudos com a canafístula (DUTRA et al., 2016), aroeira-vermelha (ROSSA et al., 2015a) e bracatinga (STUPP et al., 2015). Rossa et al. (2015a) ressaltam que entre as variáveis avaliadas, o diâmetro do coleto é um parâmetro bastante propício para avaliar a qualidade de muda, devido ao seu maior grau de relação com o IQD.

$O$ efeito significativo da interação entre os fatores avaliados (formulações e doses de Osmocote ${ }^{\circledR}$ ) foi observado para os parâmetros massa seca da parte aérea e massa seca total (Tabela 3).

Nas variáveis massa seca da parte aérea e massa seca total, o Osmocote ${ }^{\circledR}$ com tempo de liberação 7 a 8 meses, a partir da dose $5,0 \mathrm{~g} \mathrm{dm}^{-3}$, apresentou médias superiores aos osmocotes 3 a 4 meses e 5 a 6 meses (Tabela 3). Para essas variáveis, cada um dos osmocotes obtiveram médias similares a partir da dose $5,0 \mathrm{~g} \mathrm{dm}^{-3}$, não havendo diferença significativa com ganho de massa seca da parte aérea e total com aplicação de doses superiores a este 
valor (Tabela 3). Esse resultado pode ser explicado, devido ao equilíbrio na liberação gradual dessa formulação de adubo testada dentro do tempo avaliado, garantindo melhor nutrição, e favorecendo o aumento da produção de massa seca nas mudas de albízia.

Tabela 3. Valores médios de massa seca da parte aérea e massa seca total de mudas de albízia (Albizia lebbeck (L.) Benth) em resposta a diferentes formulações e doses de Osmocote ${ }^{\circledR}$ aos 120 dias.

\begin{tabular}{|c|c|c|c|c|c|}
\hline \multirow{2}{*}{$\begin{array}{l}\text { Osmocote }^{\circledR} \\
\text { (Tempo de } \\
\text { liberação) }\end{array}$} & \multicolumn{5}{|c|}{ Dose $\left(\mathrm{g} \mathrm{dm}^{-3}\right)$} \\
\hline & 0,0 & 2,5 & 5,0 & 7,5 & 10,0 \\
\hline \multicolumn{6}{|c|}{ Massa seca da parte aérea (g planta ${ }^{-1}$ ) } \\
\hline 3-4 meses & $6,6 \mathrm{aC}$ & $21,5 \mathrm{aB}$ & $40,1 \mathrm{bA}$ & $46,8 \mathrm{bA}$ & $54,7 \mathrm{bA}$ \\
\hline 5-6 meses & $6,4 \mathrm{aC}$ & $23,3 \mathrm{aB}$ & $43,4 \mathrm{bA}$ & $49,2 \mathrm{bA}$ & $57,9 \mathrm{bA}$ \\
\hline 7-8 meses & $6,9 \mathrm{aC}$ & $26,6 \mathrm{aB}$ & 51,8 aA & 56,7 aA & 66,4 aA \\
\hline \multicolumn{6}{|c|}{ Massa seca total (g planta ${ }^{-1}$ ) } \\
\hline 3-4 meses & $12,1 \mathrm{aC}$ & $30,5 \mathrm{aB}$ & $56,3 \mathrm{bA}$ & $63,3 \mathrm{bA}$ & $72,7 \mathrm{bA}$ \\
\hline 5-6 meses & $12,1 \mathrm{aC}$ & $33,9 \mathrm{aB}$ & $57,2 \mathrm{bA}$ & $64,9 \mathrm{bA}$ & $75,0 \mathrm{bA}$ \\
\hline 7-8 meses & $12,6 \mathrm{aC}$ & $38,1 \mathrm{aB}$ & $67,2 \mathrm{aA}$ & $73,2 \mathrm{aA}$ & 84,6 aA \\
\hline
\end{tabular}

Resultados semelhantes para massa seca da parte aérea foram evidenciados por Dutra et al. (2016) trabalhando com o Osmocote ${ }^{\circledR}$ no crescimento e qualidade de mudas de canafístula (Peltophorum dubium (Spreng.) Taub)) e por Serrano et al. (2006), em mudas de limoeiro "cravo" (Citrus limonia), em função da aplicação de Osmocote ${ }^{\circledR}$. O fertilizante interferiu no acúmulo de massa seca da parte aérea, sendo capaz de proporcionar excelentes ganhos médios, demonstrando assim, a grande viabilidade de uso dessa técnica de adubação na produção de mudas.

Gomes \& Paiva (2006), afirmam que o peso da massa seca da parte aérea indica a rusticidade, correlacionando essa variável diretamente com a sobrevivência, e desempenho inicial das mudas após o plantio em campo.

Segundo Almeida et al. (2005), a biomassa total exerce papel significativo no desempenho das plantas quando transferidas para o campo, influenciando na capacidade de sustentação, absorção de água e nutrientes. Serrano et al. (2010), trabalhando com a produção de mudas de mamoeiro também observou ganho em massa seca da parte aérea. A massa seca da raiz apresentou efeito significativo nas diferentes doses de Osmocote ${ }^{\circledR}$ (Tabela 4).
A massa seca de raiz das mudas de albízia apresentou médias superiores nas doses a partir de $5 \mathrm{~g} \mathrm{dm}^{-3}$, não se diferenciando estatisticamente entre si (Tabela 4).

De modo semelhante, Mendonça et al. (2004) obtiveram maiores ganhos de massa seca de raiz em mudas de maracujazeiro amarelo nas maiores doses de fertilizante de liberação controlada avaliada.

Tabela 4. Massa seca da raiz das mudas de albízia (Albizia lebbeck (L.) Benth) em resposta a diferentes doses de Osmocote ${ }^{\circledR}$ aos 120 dias.

\begin{tabular}{cc}
\hline Dose $\left(\mathbf{g ~ d m}^{-3}\right)$ & Massa seca da raiz (g planta $\left.{ }^{-1}\right)$ \\
\hline 0,0 & $5,65 \mathrm{c}$ \\
2,5 & $10,38 \mathrm{~b}$ \\
5,0 & $15,07 \mathrm{a}$ \\
7,5 & $16,21 \mathrm{a}$ \\
10,0 & $17,79 \mathrm{a}$ \\
\hline
\end{tabular}

${ }^{1}$ Médias seguidas da mesma letra minúscula na coluna não diferem entre si pelo teste Tukey a 5\% de probabilidade.

Rossa et al. (2015b) trabalhando com mudas de Eucalyptus grandis verificou que as doses de fertilizante de liberação lenta influenciaram a espécie em questão, sendo que as máximas médias em valor absoluto, para a variável massa seca da raiz corresponderam ao tratamento com $10 \mathrm{~g} \mathrm{dm}^{-3}$. Teixeira et al. (2009) também observaram ganhos significativos em mudas de dendezeiro.

Esse comportamento pode ser atribuído à resposta positiva que a albízia apresenta com a aplicação de Osmocote ${ }^{\circledR}$ em relação à produção de massa seca da raiz. A disponibilidade equilibrada dos nutrientes no período de avaliação das mudas, atendendo a demanda nutricional da espécie, refletiu no desenvolvimento e a produção de massa seca da raiz com doses maiores do adubo.

A interação entre as formulações e as doses de Osmocote ${ }^{\circledR}$ apresentou efeito significativo para a relação altura da parte aérea/massa seca da parte aérea (H/MSPA) e a relação massa seca da parte aérea/massa seca da raiz das mudas (MSPA/MSR) (Tabela 5).

A relação $H / M S P A$ pode predizer o potencial de sobrevivência da muda no campo, uma vez que quanto menor for esse índice, mais lignificada está a muda e maior a sua rusticidade (GOMES et al., 2003).

As formulações de Osmocote ${ }^{\circledR} 3$ a 4 meses e 5 a 6 meses apresentaram médias estatisticamente iguais entre suas doses, de maneira particular, a partir de 5,0 $\mathrm{g} \mathrm{dm}^{-3}$ (Tabela 5). Esse comportamento foi observado para o Osmocote ${ }^{\circledR} 7$ a 8 meses a partir da dose $2,5 \mathrm{~g} \mathrm{dm}^{-3}$, sendo 
este tratamento o que obteve as menores médias para H/MSPA, se diferenciando estatisticamente dos demais. Portanto, dentre os osmocotes e doses dos fertilizantes estudados, as mudas com maior rusticidade e potencial de sobrevivência em campo foram obtidas com o Osmocote ${ }^{\circledR} 7$ a 8 meses, na dose $2,5 \mathrm{~g} \mathrm{dm}^{-3}$.

Para a relação massa seca da parte aérea e massa seca da raiz (MSPA/MSR) os osmocotes com tempo de liberação 5 a 6 meses e 7 a 8 meses, a partir da dose 5,0 g $\mathrm{dm}^{-3}$, apresentaram médias superiores ao Osmocote ${ }^{\circledR} 3 \mathrm{a}$ 4 meses (Tabela 5). Nesta variável, cada um dos osmocotes obtiveram médias similares entre suas doses a partir de 5,0 $\mathrm{g} \mathrm{dm}^{-3}$, não havendo diferença significativa para MSPA/MSR com aplicação de doses superiores a este valor.

Tabela 5. Valores médios da relação altura e massa seca da parte aérea (H/MSPA) e relação massa seca da parte aérea e massa seca da raiz (MSPA/MSR) de mudas de albízia (Albizia lebbeck (L.) Benth) em resposta a diferentes formulações e doses de Osmocote ${ }^{\circledR}$ aos 120 dias.

\begin{tabular}{|c|c|c|c|c|c|}
\hline \multirow{2}{*}{$\begin{array}{l}\text { Osmocote }^{\circledR} \\
\text { (Tempo de } \\
\text { liberação) }\end{array}$} & \multicolumn{5}{|c|}{ Dose $\left(\mathrm{g} \mathrm{dm}^{-3}\right)$} \\
\hline & 0,0 & 2,5 & 5,0 & 7,5 & 10,0 \\
\hline \multicolumn{6}{|c|}{ H/MSPA } \\
\hline 3- 4 meses & $1,30 \mathrm{aC}$ & $0,52 \mathrm{bB}$ & $0,32 \mathrm{bA}$ & $0,29 \mathrm{aA}$ & 0,26 aA \\
\hline 5- 6 meses & $1,53 \mathrm{aC}$ & $0,53 \mathrm{bB}$ & $0,31 \mathrm{bA}$ & 0,29 aA & $0,28 a A$ \\
\hline 7- 8 meses & $1,47 \mathrm{aB}$ & $0,46 \mathrm{aA}$ & $0,27 \mathrm{aA}$ & $0,26 \mathrm{aA}$ & 0,25 aA \\
\hline \multicolumn{6}{|c|}{ MSPA/MSR } \\
\hline 3- 4 meses & $1,17 \mathrm{aC}$ & $2,37 \mathrm{aB}$ & $2,51 \mathrm{bA}$ & $2,87 \mathrm{bA}$ & $3,05 \mathrm{bA}$ \\
\hline 5- 6 meses & $1,11 \mathrm{aC}$ & $2,19 \mathrm{aB}$ & 3,18 aA & $3,21 \mathrm{aA}$ & $3,37 \mathrm{aA}$ \\
\hline 7- 8 meses & $1,20 \mathrm{aC}$ & $2,44 \mathrm{aB}$ & $3,39 \mathrm{aA}$ & 3,42 aA & 3,66 aA \\
\hline
\end{tabular}

A relação MSPA/MSR é considerada um parâmetro eficiente e seguro para expressar o padrão de qualidade das mudas (GOMES et al., 2002). Médias superiores alcançadas com os osmocotes 5 a 6 meses e 7 a 8 meses indicam uma qualidade superior nas mudas de albízia que receberam esses tratamentos.

Houve efeito significativo das diferentes doses de Osmocote $^{\circledR}$ para o Índice de Qualidade de Dickson (IQD) (Tabela 6).

O Índice de Qualidade de Dickson (IQD) apresentou as maiores médias a partir da dose $5 \mathrm{~g} \mathrm{dm}^{-3}$ de Osmocote ${ }^{\circledR}$, não se diferenciando estatisticamente entre si (Tabela 6).
Não houve efeito significativo do tipo de fertilizante para esta variável, ou seja, as mudas de albízia obtiveram o mesmo padrão de qualidade independentemente da formulação e tempo de liberação.

Tabela 6. Índice de Qualidade de Dickson das mudas de albízia (Albizia lebbeck (L.) Benth) em resposta a diferentes doses de Osmocote ${ }^{\circledR}$ aos 120 dias.

\begin{tabular}{cc}
\hline Dose $\left(\mathrm{g} \mathrm{dm}^{-3}\right)$ & Índice de Qualidade de Dickson \\
\hline 0,0 & $2,19 \mathrm{c}$ \\
2,5 & $5,28 \mathrm{~b}$ \\
5,0 & $8,53 \mathrm{a}$ \\
7,5 & $9,44 \mathrm{a}$ \\
10,0 & $10,33 \mathrm{a}$ \\
\hline
\end{tabular}

${ }^{1}$ Médias seguidas da mesma letra minúscula na coluna não diferem entre si pelo teste Tukey a 5\% de probabilidade.

Os maiores valores para essa variável foram obtidos na dose $10,0 \mathrm{~g} \mathrm{dm}^{-3}$ do adubo de liberação lenta, demonstrando respostas positivas das mudas de albízia com a aplicação do fertilizante.

O índice de qualidade de Dickson é considerado promissor por considerar as associações entre os caracteres dendrométricos e alométricos em sua fórmula matemática, pois utiliza diversos parâmetros morfológicos relevantes (ELOY et al., 2013; TRAUTENMULLER et al., 2017a). Para seu cálculo são consideradas a robustez e o equilíbrio da distribuição da biomassa na muda, ponderando os resultados de várias características importantes empregadas para avaliação da qualidade das mudas (FONSECA et al., 2002). Portanto, quanto maior o IQD, melhor é a qualidade da muda produzida.

Melo Junior et al. (2014) encontrou resposta similar no IQD com o aumento das doses de Osmocote $^{\circledR}$ na produção de mudas de mamoeiro.

Segundo Pias et al. (2013), o Osmocote ${ }^{\circledR}$ provoca menor alteração na condutividade elétrica do substrato, menor quantidade de nutrientes a serem perdidos por lixiviação no caso de irrigação excessiva, e menor risco de salinização do substrato. Por esta razão, este fertilizante é usado com grande sucesso no setor florestal, na obtenção de mudas de qualidade.

De maneira geral, os osmocotes estudados apresentaram as maiores médias para todas as variáveis analisadas com a dose $10,0 \mathrm{~g} \mathrm{dm}^{-3}$. Entretanto, a dose 5,0 $\mathrm{g} \mathrm{dm}^{-3}$ não se diferenciou estatisticamente da dose máxima, significando que a adoção desse valor na adubação traria ganhos semelhantes para as mudas e economia de custos com o fertilizante na produção.

Cabe ressaltar que o Osmocote ${ }^{\circledR} 7$ a 8 meses se 
destacou frente aos demais, com valores superiores para diversos parâmetros.

Diante disso, a diferença de valores entre as doses recomendadas para cada espécie florestal reforça a ideia da necessidade de estudos particulares, com o intuito de buscar o melhor desenvolvimento das mudas e a otimização no uso da técnica, sendo difícil a realização de uma recomendação padrão (DUTRA et al., 2016).

\section{CONCLUSÕES}

O Osmocote ${ }^{\circledR}$ com tempo de liberação de 7 a 8 meses proporciona maior produção de massa seca às mudas de Albizia lebbeck (L.) Benth.

A maior dose estudada $\left(10,0 \mathrm{~g} \mathrm{dm}^{-3}\right)$ permitiu as maiores médias para as variáveis analisadas, entretanto, os valores foram semelhantes à dose $5,0 \mathrm{~g} \mathrm{dm}^{-3}$, o que permite uma economia nos custos de produção.

Os adubos Osmocote ${ }^{\circledR}$ com tempo de liberação 5 a 6 meses, 7 a 8 meses e 3 a 4 meses são recomendados para a produção de mudas de albízia.

\section{AGRADECIMENTOS}

Ao Instituto Federal do Norte de Minas Gerais (IFNMG), Câmpus Salinas. À CAPES/Prodoutoral pela concessão de bolsas ao segundo e terceiro autores. À Coordenação de Aperfeiçoamento de Pessoal de Nível Superior - Brasil (CAPES) - Código de Financiamento 001.

\section{REFERÊNCIAS}

ALMEIDA, S.L. et al. Crescimento de mudas de Jacaranda puberula Cham. em viveiro submetidas a diferentes níveis de luminosidade. Revista Ciência Florestal, v.15, n.3, p.323-329, 2005.

ALMEIDA, U.O. et al. Environment and slow-release fertilizer in the production of Euterpe precatoria seedlings. Pesquisa Agropecuária Tropical, v.48, n.4, p.382-389, 2018.

CARVALHO, C.M.; SILVA, C.R. Determinação das propriedades físicas de substrato. Botucatu: Faculdade de Ciências Agronômicas: Universidade Estadual Paulista, 1992.

CHEN, S. et al. Preparation and characterization of slow-release fertilizer encapsulated by biochar-based waterborne copolymers. Science of The Total Environment, v.615, n.15, p.431-437, 2018.

DICKSON, A. et al. Qualitty appraisal of white spruce and white pine seedling stock in nurseries. Forestry Chronicle, v.36, p.1013, 1960.

DUTRA, A.S.; MEDEIROS FILHO, S. Dormência e germinação de sementes de albizia (Albizia lebbeck (L.) Benth). Revista Ciência Agronômica, v.40, n.3, p.427-429, 2009.

DUTRA, A.S. et al. Dormência, substrato e temperatura para germinação de sementes de albizia (Albizia lebbeck (L.)). Revista Ciência Agronômica, v.38, n.3, p.291-296, 2007.

DUTRA, T.R. et al. Fertilizante de liberação lenta no crescimento e qualidade de mudas de canafístula (Peltophorum dubium). Floresta, v.46, n.4, p.491-498, 2016.

ELLI, E.F. et al. Osmocote ${ }^{\bullet}$ no desenvolvimento e comportamento fisiológico de mudas de pitangueira. Comicata Scientiae, v.4, n.4, p.337-384, 2013.

ELOY, E. et al. Avaliação da qualidade de mudas de Eucalyptus grandis utilizando parâmetros morfológicos. Floresta, v.43, n.3, p.373-384, 2013.

EMBRAPA. Centro Nacional de Pesquisa de Solos. Manual de métodos de análise de solos. 2 ed. Rio de Janeiro: EMBRAPA, 1997.

FERREIRA, E.B. et al. ExpDes.pt: experimental designs package $\mathbf{R}$ package version (1.1.2). 2013. Disponível em: http://cran.rproject.org/web/packages/ExpDes/index.html.

FONSECA, E.P. et al. Padrão de qualidade de mudas de Trema micrantha (L.) Blume, produzidas sobre diferentes períodos de sombreamento. Revista Árvore, v.26, n.4, p.515-523, 2002.

GIBSON, E.L. et al. Controlled-Release Fertilizer on Growth of Melanoxylon brauna Schott Seedlings. Floresta Ambiente, v.26, p.1-7, 2019.

GOMES, J.M. et al. Parâmetros morfológicos na avaliação da qualidade de mudas de Eucalyptus grandis. Revista Árvore, v.26, n.6, p.655-664, 2002.

GOMES, J.M. et al. Crescimento de mudas de Eucalyptus grandis em diferentes tamanhos de tubetes e fertilização N-P-K. Revista Árvore, v.23, p.113-127, 2003.

GOMES, J.M.; PAIVA, H.N. Viveiros Florestais: propagação sexuada. 3.ed. Viçosa: UFV, 2006.

MARANA, J.P. et al. Índices de qualidade e crescimento de mudas de café produzidas em tubetes. Ciência Rural, v.38, n.1, p.39-45, 2008.

MASSAD, M.D. et al. Desenvolvimento de mudas de flamboyant e ipê-mirim em resposta a diferentes doses de Osmocote ${ }^{\circledR}$. Revista Agropecuária Científica no Semiárido, v.12, n.1, p.8392, 2016.

MELO JÚNIOR, J.C.F. et al. Depleção de água no substrato e doses de fertilizante Osmocote ${ }^{\circledR}$ na formação de mudas de mamoeiro. Comicata Scientiae, v.5, n.4, p.499-508, 2014.

MENDONÇA, V. et al. Osmocote e substratos alternativos na produção de mudas de maracujazeiro-amarelo. Revista Ciência e Agrotecnologia, v.28, n.4, p.799-806, 2004.

MENEGATTI, R.D. et al. Fertilizante de liberação lenta no 
desenvolvimento inicial de Aspidosperma parvifolium A. DC. Scientia Agraria Paranaensis, v.16, p.45-49, 2017.

MENEZES, E.S. et al. Crescimento de mudas de Peltophorum dubium (Spreng.) Taub. sob interferência de plantas espontâneas e forrageiras. BIOFIX Scientific Journal, v.4, n.2, p.153-159, 2019.

MEWS, C.L. et al. Efeito do hidrogel e ureia na produção de mudas de Handroanthus ochraceus (Cham.) Mattos. Floresta e Ambiente, v.22, n.1, p.107-116, 2015.

PIAS, C.H.O. et al. Doses de fertilizante de liberação controlada no índice de clorofila e na produção de mudas de grápia. Pesquisa Florestal Brasileira, v.33, n.73, p.19-26, 2013.

R CORE TEAM. R: A language and environment for statistical computing. Vienna: R Foundation for Statistical Computing, 2015. Disponível em: http://www.R-project.org

ROSA, T.L.M. et al. Controlled release fertilizer in the growth of Moringa oleifera Lam. Seedlings. Floresta, v.48, n.3, p.303-310, 2018.

ROSSA, U.B. et al. Fertilizante de liberação lenta no desenvolvimento de mudas de Anadenanthera peregrina (L.) Speg. (angico-vermelho) e Schinus terebinthifolius Raddi (aroeira-vermelha). Ciência Florestal, v.25, n.4, p.841-852, 2015a.

ROSSA, U.B. et al. Fertilizante de liberação lenta no desenvolvimento de mudas de Eucalyptus grandis. Floresta, v.45, n.1, p.85-96, 2015b.

SERRANO, L.A.L. et al. Sistema de blocos prensados e doses de adubo de liberação lenta na formação de porta-enxerto cítrico. Ciência Rural, v.36, n.2, p.441-447, 2006.

SERRANO, L.A.L. et al. Adubo de liberação lenta na produção de mudas de mamoeiro. Revista Brasileira Fruticultura, v.32, n.3, p.874-883, 2010.

SOMAVILLA, A. et al. Avaliações morfológicas de mudas de Cedro australiano submetidas a diferentes doses do fertilizante Osmocote plus ${ }^{\circ}$. Comunicata Scientiae, v.5, n.4, p.493-498, 2014.

STUPP, A.M. et al. Crescimento de mudas de Mimosa scabrella Benth em função de diferentes tamanhos de recipientes e doses de fertilizante. Ecologia e Nutrição Florestal, v.3, n.2, p.40-47, 2015.

TEIXEIRA, P.C. et al. Influência da disposição dos tubetes e da aplicação de fertilizantes de liberação lenta, durante o préviveiro, no crescimento de mudas de dendezeiro (Elaeis guineensis Jacq.). Ciência Florestal, v.19, n.2, p.157-168, 2009.

TOLEDO, L.O. et al. Análise multivariada de atributos pedológicos e fitossociológicos aplicada na caracterização de ambientes de cerrado no norte de Minas Gerais. Revista Árvore, v.33, n.5, p.957-968, 2009.

TRAUTENMULLER, J.W. et al. Avaliação de plantas de Cordia americana em viveiro utilizando caracteres morfológicos. BIOFIX
Scientific Journal, v.2, n.2, p.65-70, 2017a.

TRAUTENMULLER, J.W. et al. Crescimento de área foliar e índice de área foliar de mudas de Cordia americana em diferentes formas de manejo. BIOFIX Scientific Journal, v.2, n.2, p.60-64, 2017b.

Recebido em 30-08-2019

Aceito em 03-12-2019 